\title{
On the Stability and Strong Convergence for Jungck- Agarwal et al. Iteration Procedure
}

\author{
Renu Chugh and Sanjay Kumar \\ Department of Mathematics \\ Maharshi Dayanand University \\ ROHTAK, INDIA
}

\begin{abstract}
In this paper we introduce the Jungck-Agarwal et al. iteration procedure and obtain strong convergence as well as stability results for a pair of non-self mappings. The results obtained are generalization of some existing results in the literature. In addition, we show that the rate of convergence of this newly defined iteration procedure is better than Jungck- Mann, Jungck-Ishikawa and Jungck- Noor iteration procedures.
\end{abstract}

\section{General Terms}

Computational Mathematics.

\section{Keywords}

Jungck-Ishikawa iteration, Jungck-Agarwal et al. iteration , Jungck-Noor iteration, Jungck-Mann iteration, Stability.

\section{INTRODUCTION}

Let (E, d) be a complete metric space, T be $a$ self map of E. Suppose that $F_{T}=\{p \in E$ s.t $T p=p\} \quad$ is the set of fixed points of $\mathrm{T}$ in $\mathrm{E}$. In metric space we have several iterative processes that have been defined by many researchers to approximate the fixed points of different operators. Some of them are the following:

Let $\left\{x_{n}\right\}_{n=0}^{\infty} \subset E$ be the sequence generated by an iteration procedure involving the operator $\mathrm{T}$, that is,

$x_{n+1}=f\left(T, x_{n}\right) \quad n=0,1,2$

where $x_{0} \in E$, be the initial approximation and $\mathrm{f}$ is some function. If in (1.1)

$f\left(T, x_{n}\right)=T x_{n}$

Then we have Picard iteration process which has been used to approximate the fixed point of mappings satisfying the inequality:

$d(T x, T y) \leq a d(x, y), \forall x, y \in E$ and $a \in[0,1)$

Condition (1.3) is called Banach contraction condition and operator which satisfies the condition is called strict contraction. Also condition (1.3) is significant in Banach Fixed point theorem [2].

Let $x_{0} \in E$, be the initial approximation, where $\mathrm{E}$ is any Banach space, then

In 1953, W.R. Mann defined the Mann iteration [10] as

$x_{n+1}=\left(1-\alpha_{n}\right) x_{n}+T x_{n}$
Where $\left\{\alpha_{n}\right\}_{n=0}^{\infty}$ is real sequence in $[0,1]$ satisfying $\sum_{k=0}^{\infty} \alpha_{k}=\infty$

For $x_{0} \in E$, Ishikawa Iteration [7] (S. Ishikawa 1974)

$\left\{x_{n}\right\}_{n=0}^{\infty}$ is defined as

$x_{n+1}=\left(1-\alpha_{n}\right) x_{n}+\alpha_{n} T y_{n}$

$y_{n}=\left(1-\beta_{n}\right) x_{n}+\beta_{n} T x_{n}$

Where $\left\{\alpha_{n}\right\}_{n=0}^{\infty}$ and $\left\{\beta_{n}\right\}_{n=0}^{\infty}$ are real sequences in $[0,1]$ satisfying $\sum_{k=0}^{\infty} \alpha_{k}=\infty$.Observe that if $\beta_{n}=0$ for each $n$, then the Ishikawa iteration process (1.5) reduces to the Mann iteration scheme (1.4).

For $x_{0} \in E$ such that $\left\{x_{n}\right\}_{n=0}^{\infty}$, the Noor three step iteration scheme [11] is defined as

$x_{n+1}=\left(1-\alpha_{n}\right) x_{n}+T y_{n}$

$y_{n}=\left(1-\beta_{n}\right) x_{n}+T z_{n}$

$z_{n}=\left(1-\gamma_{n}\right) x_{n}+T x_{n}$

where $\left\{\alpha_{n}\right\}_{n=0}^{\infty},\left\{\beta_{n}\right\}_{n=0}^{\infty}$ and $\left\{\gamma_{n}\right\}_{n=0}^{\infty}$ are real sequences in $[0,1]$ satisfying $\sum_{k=0}^{\infty} \alpha_{k}=\infty$

If $\gamma_{\mathrm{n}}=0$ then Noor iteration process (1.6) reduces to Ishikawa Iteration scheme (1.5)

\section{Agarwal Iteration Process}

In 2007, Agarwal et al. defined the Agarwal et al. iterative scheme [1] as

$\mathrm{x}_{\mathrm{n}+1}=\left(1-\alpha_{\mathrm{n}}\right) \mathrm{Tx}_{\mathrm{n}}+\alpha_{\mathrm{n}} \mathrm{Ty}_{\mathrm{n}}$

$\mathrm{y}_{\mathrm{n}}=\left(1-\beta_{\mathrm{n}}\right) \mathrm{x}_{\mathrm{n}}+\beta_{\mathrm{n}} T \mathrm{x}_{\mathrm{n}}$

Where $\left\{\alpha_{n}\right\}$ and $\left\{\beta_{n}\right\}$ are sequences of positive numbers in $[0,1]$ satisfying $\quad \sum_{k=0}^{\infty} \alpha_{k}=\infty$

Zemfirescu [25] established a nice generalization of the Banach fixed point theorem as

Let $\mathrm{T}$ be a self map, there exists real numbers $\alpha, \beta, \gamma$ satisfying $0 \leq \alpha<1,0 \leq \beta<\frac{1}{2}, 0 \leq \gamma<\frac{1}{2}$ respectively such that for each $x, y \in E$, at least one of the following is true: 


$$
\begin{aligned}
& \left(z_{1}\right) d(T x, T y) \leq \alpha d(x, y) \\
& \left(z_{2}\right) d(T x, T y) \leq \beta[d(x, T x)+d(y, T y)] \\
& \left(z_{3}\right) d(T x, T y) \leq \gamma[d(x, T y)+d(y, T x)]
\end{aligned}
$$

Then the mapping $\mathrm{T}$ satisfying (1.8) is called Zamfirescu contraction.

\section{Remark}

Mapping which satisfy $\left(z_{2}\right)$ is called a Kannan mapping.While the mapping satisfying $\left(\mathrm{z}_{3}\right)$ is called Chatterjea operator.

The contractive condition (1.8) implies

$\|T x-T y\| \leq 2 \delta\|x-T x\|+\delta\|x-y\|, \forall x, y \in E$,

Where

$\delta=\max \left\{\alpha, \frac{\beta}{1-\beta}, \frac{\gamma}{1-\gamma}\right\}, 0 \leq \delta<1$.

Rhoades [21, 22] used condition (1.8) in some convergence results for Mann and Ishikawa iteration process in a uniformly convex Banach space. Berinde [3] extended (1.8) to a new class of operators to an arbitrary Banach space and proved that this class is wider than class of Zamfirescu operators.

Several stability results are established by various authors in literature due to its increasing importance in computational mathematics. Some of the various authors whose contribution are much important in the study of stability of fixed point iteration process are Ostrowski [18], Harder and Hicks [6], Rhoades[20, 21], Osillike[16], Osilike and Udomene[15] ,Jachymski [8] ,Berinde [4, 5] and Singh et al. [24]. The summability theory of infinite matrices are used by Harder and Hicks [6], Rhoades [22], Osilike[23], and Singh et al.[24] to prove different stability results for certain contractive conditions. The first stability result on $\mathrm{T}$ - stable mappings was due to Ostrowski[18] for the stability of Picard iteration using Banach contraction condition. Osilike and Udomene[15] introduced a shorter method for stability results and has also been employed by Berinde[4] ,Imoru and Olatinwo[12],Olatinwo et al. [13] and some others.

\section{Preliminaries}

In 1976, Jungck introduced the Jungck iteration scheme [9] as

Let $\mathrm{S}, \mathrm{T}$ be arbitrary operators on any set $\mathrm{Y}$ with values in $\mathrm{E}$ such that $\mathrm{T}(\mathrm{Y}) \subseteq \mathrm{S}(\mathrm{Y}), \mathrm{S}(\mathrm{Y})$ is complete subspace of $\mathrm{E}$. for any $x_{0} \in Y$, the sequence $\left\{S x_{n}\right\}_{n=0}^{\infty}$ defined as

$S x_{n+1}=T x_{n} \quad n=0,1 \ldots \ldots$

For S=Id (2.1) becomes Picard iteration

Singh et al [24] in 2005 introduce the following iteration to establish some stability result as:

$S x_{n+1}=\left(1-\alpha_{n}\right) S x_{n}+\alpha_{n} T x_{n} \quad \mathrm{n}=0,1,2 \ldots \ldots$

Where $\left\{\alpha_{n}\right\}_{n=0}^{\infty}$ is a sequence in [0,1], called Jungck-Mann iteration process.

Jungck [9] established that the maps $\mathrm{S}$ and $\mathrm{T}$ satisfying

$$
d(T x, T y) \leq k d(S x, S y), \quad 0 \leq k<1 \forall x, y \in E
$$

has a unique common fixed point. For $\mathrm{Y}=\mathrm{E}$ and $\mathrm{S}=\mathrm{Id}$ above mapping becomes well known contraction map.
Olatinwo \& Imoru in 2008 for $x_{0} \in Y$ defined $\left\{S x_{n}\right\}_{n=1}^{\infty}$ as

$$
\begin{aligned}
& S x_{n+1}=\left(1-\alpha_{n}\right) S x_{n}+\alpha_{n} T x_{n} \\
& S y_{n}=\left(1-\beta_{n}\right) S x_{n}+\beta_{n} T x_{n}
\end{aligned}
$$

Called Jungck-Ishikawa Iteration scheme [13]

Where $\left\{\alpha_{n}\right\}_{n=0}^{\infty}$ and $\left\{\beta_{n}\right\}_{n=0}^{\infty}$ are real sequences in $[0,1]$ satisfying $\sum_{k=0}^{\infty} \alpha_{k}=\infty$

Oltinwo in 2008 define Jungck-Noor iteration [14] process as

Let $x_{o} \in Y$ then $\left\{S x_{n}\right\}_{n=1}^{\infty}$ is defined by

$$
\begin{aligned}
& S x_{n+1}=\left(1-\alpha_{n}\right) S x_{n}+\alpha T y_{n} \\
& S y_{n}=\left(1-\beta_{n}\right) S x_{n}+\beta_{n} T x_{n} \\
& S z_{n}=\left(1-\gamma_{n}\right) S x_{n}+\gamma_{n} T x_{n}
\end{aligned}
$$

where $\left\{\alpha_{n}\right\}_{n=0}^{\infty},\left\{\beta_{n}\right\}_{n=0}^{\infty}$ and $\left\{\gamma_{n}\right\}_{n=0}^{\infty}$ are real sequences in $[0,1]$ satisfying $\sum_{k=0}^{\infty} \alpha_{k}=\infty$

A more general contractive condition than (1.9) was used by Oltinwo[13] to prove stability and strong convergence in Jungck-Ishikawa iteration process.

(a) There exists a real number $a \in[0,1)$ and a monotone increasing function $\varphi: R^{+} \rightarrow R^{+}$,

such that $\varphi(0)=0$ and $\forall x, y \in Y$, we have

$$
\|T x-T y\| \leq \varphi(\|S x-T x\|)+a\|S x-S y\|
$$

(b) There exist a real number $\mathrm{M} \geq 0, a \in[0,1)$ and a monotone increasing function $\varphi: R^{+} \rightarrow R^{+}$such that $\varphi(0)=0$ and $\forall \mathrm{x}, \mathrm{y} \in \mathrm{Y}$, we have

$\|T x-T y\| \leq \frac{\varphi(\|S x-T x\|)+a\|S x-S y\|}{1+M\|S x-T x\|}$

In this paper, we define following new Jungck-Agarwal et al. iteration process and use it to approximate the common fixed point by using contractive condition $(2.5,2.6)$

$$
\begin{aligned}
& S x_{n+1}=\left(1-\alpha_{n}\right) T x_{n}+\alpha_{n} T y_{n} \\
& S y_{n}=\left(1-\beta_{n}\right) S x_{n}+\beta_{n} T x_{n}
\end{aligned}
$$

Where $\left\{\alpha_{n}\right\}_{n=0}^{\infty}$ and $\left\{\beta_{n}\right\}_{n=0}^{\infty}$ are real sequences in $[0,1]$ satisfying $\sum_{k=0}^{\infty} \alpha_{k}=\infty$

We use following definition and lemma:

Definition 2.1. [12] Let $\mathrm{S}, \mathrm{T}: \mathrm{Y} \rightarrow \mathrm{X}$ such that $T(Y) \subseteq$ $S(Y)$ and $z$ a coincidence point of $S$ and $T$, that is, $S z=T z=p$ (say). For any $x_{0} \in Y$. Let the sequence $\left\{S x_{n}\right\}_{n=1}^{\infty}$ generated by the iteration procedure (2.7) converges to $\mathrm{p}$. Let $\left\{S y_{n}\right\}_{n=0}^{\infty} \subseteq E$ be an arbitrary sequence, and set $\varepsilon_{n}=d\left(S y_{n+1}, f\left(T, y_{n}\right)\right), \quad n=0,1,2 \ldots$. Then, the iteration procedure (2.7) will be called (S, T)-stable if and only if $\lim _{n \rightarrow \infty} \varepsilon_{n}=0$ implies that $\lim _{n \rightarrow \infty} S\left(y_{n}\right)=p$. 
Lemma 2.1. (Berinde [3]): If $\delta$ is a real number such that $0 \leq \delta<1$, and $\left\{\varepsilon_{n}\right\}_{n=0}^{\infty}$ is a sequence of positive numbers such that $\lim _{n \rightarrow \infty} \varepsilon_{n}=0$, then for any sequence of positive numbers $\left\{u_{n}\right\}_{n=0}^{\infty}$ satisfying

$u_{n+1} \leq \delta u_{n}+\varepsilon_{n}, \quad n=0,1,2 \ldots .$.

We have $\lim _{n \rightarrow \infty} u_{n}=0$

In this paper we shall prove convergence results for JungckAgarwal et al. iteration process for arbitrary Banach space and stability result for nonlinear mappings in normed linear space by using contractive condition $(2.5,2.6)$. Also by using C++ we shall prove that Jungck-Agarwal et al. iteration scheme converges faster than other jungck iteration scheme.

\section{STABILITY RESULT IN NORMED LINEAR SPACE.}

Theorem 3.1. Let $(E,\|\|)$ be a normed linear space and $\mathrm{Y}$ an arbitrary set. Suppose that $\mathrm{S}, \mathrm{T}: \mathrm{Y} \rightarrow \mathrm{E}$ are nonself operators such that $\mathrm{T}(\mathrm{Y}) \subseteq \mathrm{S}(\mathrm{Y})$. $\mathrm{S}(\mathrm{Y})$ a complete subspace of $\mathrm{E}$ and $\mathrm{S}$ is an injective operator. Let $\mathrm{z}$ be a coincidence point of $\mathrm{S}$ and $\mathrm{T}$ (that is, $\mathrm{Sz}=\mathrm{Tz}=\mathrm{p}$ ). Suppose that $\mathrm{S}$ and $\mathrm{T}$ satisfy condition $(2.5,2.6)$. Let $\varphi: \mathrm{R}^{+} \rightarrow \mathrm{R}^{+}$be monotone increasing function such that $\varphi(0)=0$. For $x_{0} \in \mathrm{Y}$, let $\left\{S x_{n}\right\}_{n=0}^{\infty}$ be Jungck- Agarwal et al. iteration process defined by (2.7) converging to $\mathrm{p}$, where $\left\{\alpha_{n}\right\}_{n=0}^{\infty}$ and $\left\{\beta_{n}\right\}_{n=0}^{\infty}$ are sequences in $[0,1]$, such that $0<\alpha \leq \alpha_{\mathrm{n}}$ and $0<\beta \leq \beta_{\mathrm{n}}$. Then, the Jungck-Agarwal et al. iteration process is $(\mathrm{S}, \mathrm{T})$-stable.

Proof: Suppose that $\left\{S x_{n}\right\}_{n=0}^{\infty} \subset \mathrm{E}, \varepsilon_{n}=$

$\left\|S x_{n+1}-\left(1-\alpha_{n}\right) T x_{n}-\alpha_{n} T y_{n}\right\|, \mathrm{n}==0,1,2 \ldots$,

and let $\lim _{n \rightarrow \infty} \varepsilon_{n}=0$. Then, we shall establish that

$\lim S x_{n}=p$, using the contractive condition $(2.5,2.6)$ and the

triangle inequality:

$$
\begin{aligned}
& \left\|S x_{n+1}-p\right\| \leq\left\|S x_{n+1}-\left(1-\alpha_{n}\right) T x_{n}-\alpha_{n} T y_{n}\right\|+ \\
& \left\|\left(1-\alpha_{n}\right) T x_{n}+\alpha_{n} T y_{n}-\left(1-\alpha_{n}+\alpha_{n}\right) p\right\| \\
& \leq\left\|S x_{n+1}-\left(1-\alpha_{n}\right) T x_{n}-\alpha_{n} T y_{n}\right\| \\
& +\left(1-\alpha_{n}\right)\left\|T x_{n}-p\right\|+\alpha_{n}\left\|T y_{n}-p\right\| \\
& \leq \varepsilon_{n}+\left(1-\alpha_{n}\right) a\left\|S x_{n}-p\right\|+\alpha_{n} a\left\|S y_{n}-p\right\| \\
& \left\|S y_{n}-p\right\|=\left\|\left(1-\beta_{n}\right) S x_{n}+\beta_{n} T x_{n}+\left(1-\beta_{n}+\beta_{n}\right) p\right\| \\
& \leq\left(1-\beta_{n}\right)\left\|S x_{n}-p\right\|+\beta_{n}\left\|T x_{n}-p\right\| \\
& \leq\left(1-\beta_{n}\right)\left\|S x_{n}-p\right\|+a \beta_{n}\left\|S x_{n}-p\right\| \\
& \leq\left(1-\beta_{n}+a \beta_{n}\right)\left\|S x_{n}-p\right\|
\end{aligned}
$$$$
\leq \varepsilon_{n}+\left(1-\alpha_{n}\right)\left[\frac{\varphi(\|S z-T z\|)+a\left\|S z-S x_{n}\right\|}{1+M\|S z-T z\|}\right]+\alpha_{n}\left[\frac{\varphi(\|S z-T z\|)+a \| S z-S y_{n}}{1+M\|S z-T z\|}\right.
$$

Using (3.2) in (3.1) yields

$$
\begin{aligned}
\| S x_{n+1} & -p \| \\
& \leq\left[a\left(1-\alpha_{n}\right)+\alpha_{n} a\left(1-\beta_{n}+a \beta_{n}\right)\right]\left\|S x_{n}-p\right\|+\varepsilon_{n} \\
& \leq a\left[1-(1-a) \alpha_{n} \beta_{n}\right]\left\|S x_{n}-p\right\|+\varepsilon_{n} \\
& \leq\left[1-(1-a) \alpha_{n}\right]\left\|S x_{n}-p\right\|+\varepsilon_{n} \\
& \leq[1-(1-a) \alpha]\left\|S x_{n}-p\right\|+\varepsilon_{n}
\end{aligned}
$$

Since $0 \leq 1-(1-a) \alpha<1$, using Lemma 2.1 in (3.3) yields

$\lim _{n \rightarrow \infty}\left\|S x_{n}-p\right\|=0$, that is, $\lim _{n \rightarrow \infty} S x_{n}=p$

Conversely, let $\lim _{n \rightarrow \infty} S x_{n}=p$. Then, by using the triangle

inequality and contractive definition, we have the following:

$$
\begin{aligned}
\varepsilon_{n} & =\left\|S x_{n+1}-\left(1-\alpha_{n}\right) T x_{n}-\alpha_{n} T y_{n}\right\| \\
& \leq\left\|S x_{n+1}-p\right\|+\left\|\left(1-\alpha_{n}+\alpha_{n}\right) p-\left(1-\alpha_{n}\right) T x_{n}-\alpha_{n} T y_{n}\right\| \\
& \leq\left\|S x_{n+1}-p\right\|+\left(1-\alpha_{n}\right)\left\|T x_{n}-p\right\|+\alpha_{n}\left\|T y_{n}-p\right\| \\
& \leq\left\|S x_{n+1}-p\right\|+\left(1-\alpha_{n}\right) a\left\|S x_{n}-p\right\|+\alpha_{n} a\left\|S y_{n}-p\right\|
\end{aligned}
$$

Again, by the contractive condition(2.6), we get

$$
\begin{aligned}
\left\|S y_{n}-p\right\| & =\left\|\left(1-\beta_{n}+\beta_{n}\right) p-\left(1-\beta_{n}\right) S x_{n}-\beta_{n} T x_{n}\right\| \\
& =\left\|\left(1-\beta_{n}\right)\left(p-S x_{n}\right)+\beta_{n}\left(p-T x_{n}\right)\right\| \\
& \leq\left(1-\beta_{n}\right)\left\|p-S x_{n}\right\|+\beta_{n}\left\|p-T x_{n}\right\| \\
& =\left(1-\beta_{n}\right)\left\|p-S x_{n}\right\|+\beta_{n}\left\|T z-T x_{n}\right\| \\
& \leq\left(1-\beta_{n}+a \beta_{n}\right)\left\|p-S x_{n}\right\|
\end{aligned}
$$

Using (3.5) in (3.4), we obtain

$$
\begin{aligned}
\varepsilon_{n} & \leq\left\|S x_{n+1}-p\right\|+a\left[1-(1-a) \alpha_{n} \beta_{n}\right]\left\|S x_{n}-p\right\| \\
& \leq\left\|S x_{n+1}-p\right\|+\left[1-(1-a) \alpha_{n}\right]\left\|S x_{n}-p\right\| \\
& \leq\left\|S x_{n+1}-p\right\|+[1-(1-a) \alpha]\left\|S x_{n}-p\right\| \rightarrow 0 \text { as } n \rightarrow \infty
\end{aligned}
$$

Hence, the iteration process defined in (2.7) is stable with respect to the pair $(\mathrm{S}, \mathrm{T})$.

\section{STRONG CONVERGENCE IN AN ARBITRARY BANACH SPACE}

Theorem 4.1: Let $(E,\|\|)$ be arbitrary Banach space and $\mathrm{Y}$ an arbitrary set. Suppose that $\mathrm{S}, \mathrm{T}: \mathrm{Y} \rightarrow \mathrm{E}$ are nonself operators such that $\mathrm{T}(\mathrm{Y}) \subseteq \mathrm{S}(\mathrm{Y})$. $\mathrm{S}(\mathrm{Y})$ a complete subspace of $\mathrm{E}$ and $\mathrm{S}$ is an injective operator. Let $\mathrm{z}$ be a coincidence point of $\mathrm{S}$ and $\mathrm{T}$ (that is, $\mathrm{Sz}=\mathrm{Tz}=\mathrm{p}$ ). Suppose that $\mathrm{S}$ and $\mathrm{T}$ satisfy condition $(2.5,2.6)$. Let $\varphi: \mathrm{R}^{+} \rightarrow \mathrm{R}^{+}$be monotone increasing function such that $\varphi(0)=0$. For $x_{0} \in Y$, let $\left\{S x_{n}\right\}_{n=0}^{\infty}$ be Jungck- Agarwal et al. iteration process defined by (2.7) converging to $\mathrm{p}$, where $\left\{\alpha_{n}\right\}_{n=0}^{\infty}$ and $\left\{\beta_{n}\right\}_{n=0}^{\infty}$ are sequences in $[0,1]$, such that $\sum_{k=0}^{\infty} \alpha_{k}=\infty$. Then, $\left\{S x_{n}\right\}_{n=0}^{\infty}$ converges strongly to $\mathrm{p}$.

Proof: Let $\mathrm{C}(\mathrm{S}, \mathrm{T})$ be the set of the coincidence points of $\mathrm{S}$ and $\mathrm{T}$. We shall now use condition (2.5) to establish that $\mathrm{S}$ and $\mathrm{T}$ have a unique coincidence point $\mathrm{z}$ (i.e. $\mathrm{Sz}=\mathrm{Tz}=\mathrm{p}$ (say)): Suppose that there exists $z_{1}, z_{2} \in C(S, T)$ such that $S z_{1}=T z_{1}=p_{1}$ and $\mathrm{Sz}_{2}=\mathrm{Tz}_{2}=\mathrm{p}_{2}$. If $\mathrm{p}_{1}=\mathrm{p}_{2}$, then $\mathrm{Sz}_{1=} \mathrm{Sz}_{2}$ and since $\mathrm{S}$ is 
injective, it follows that $\mathrm{z}_{1}=\mathrm{z}_{2}$.If $\mathrm{p}_{1} \neq \mathrm{p}_{2}$, then we have by contractiveness condition (2.5) for $\mathrm{S}$ and $\mathrm{T}$ that

$$
\begin{aligned}
0<\left\|p_{1}-p_{2}\right\| & =\left\|T z_{1}-T z_{2}\right\| \leq \varphi\left(\left\|S z_{1}-T z_{2}\right\|\right)+a\left\|S z_{1}-S z_{2}\right\| \\
& =a\left\|p_{1}-p_{2}\right\|
\end{aligned}
$$

Which leads to $(1-a)\left\|p_{1}-p_{2}\right\| \leq 0$, from which it follows that 1 -a $>0$ since $\mathrm{a} \in[0,1)$, but $\left\|p_{1}-p_{2}\right\| \leq 0$, which is a contradiction since norm is nonnegative. Therefore, we have that $\left\|p_{1}-p_{2}\right\|=0$, that is, $\mathrm{p}_{1}=\mathrm{p}_{2}=\mathrm{p}$. Since $\mathrm{p}_{1}=\mathrm{p}_{2}$, then we have that $\mathrm{p}_{1}=\mathrm{Sz}_{1}=\mathrm{Tz}_{1}==\mathrm{Sz}_{2}=\mathrm{Tz}_{2}=\mathrm{p}_{2}$, leading to $\mathrm{Sz}_{1}=\mathrm{Sz}_{2} \Rightarrow \mathrm{z}_{1}=\mathrm{z}_{2}=\mathrm{z}$ (since $S$ is injective). Hence, $z \in C(S, T)$, that is, $z$ is unique coincidence point of $\mathrm{S}$ and $\mathrm{T}$.

We now prove that $\left\{S x_{n}\right\}_{n=0}^{\infty}$ converges strongly to $\mathrm{p}$ (where $\mathrm{Sz}=\mathrm{Tz}=\mathrm{p}$ ) using again, condition $(2.5,2.6)$. Therefore, we have

$$
\begin{aligned}
\left\|S x_{n+1}-p\right\| & =\left\|\left(1-\alpha_{n}\right) T x_{n}+\alpha_{n} T y_{n}-\left(1-\alpha_{n}+\alpha_{n}\right) p\right\| \\
\leq & \left(1-\alpha_{n}\right)\left\|p-T x_{n}\right\|+\alpha_{n}\left\|p-T y_{n}\right\| \\
= & \left(1-\alpha_{n}\right)\left\|T z-T x_{n}\right\|+\alpha_{n}\left\|T z-T y_{n}\right\| \\
\leq & \left(1-\alpha_{n}\right)\left[\frac{\left.\varphi(\|S z-T z\|)+a\left\|S z-S x_{n}\right\|_{1}\right]}{1+M\|S z-T z\|}\right. \\
& +\alpha_{n}\left[\frac{\left.\varphi(\|S z-T z\|)+a\left\|S z-S y_{n}\right\|_{1}\right]}{1+M\|S z-T z\|}\right. \\
\leq & \left(1-\alpha_{n}\right) a\left\|S x_{n}-p\right\|+\alpha_{n} a\left\|S y_{n}-p\right\| \\
\left\|S y_{n}-p\right\| \leq & \left\|\left(1-\beta_{n}\right) S x_{n}+\beta_{n} T x_{n}+\right\| \\
\leq & \left\|\left(1-\beta_{n}\right) S x_{n}+\beta_{n}\left(T z-T x_{n}\right)-\left(1+\beta_{n}-\beta_{n}\right) p\right\| \\
\leq & \left(1-\beta_{n}\right)\left\|S x_{n}-p\right\|+\beta_{n}\left\|T x_{n}-p\right\| \\
\leq & \left(1-\beta_{n}\right)\left\|S x_{n}-p\right\| \\
& +\beta_{n}\left[\frac{\left.\varphi(\|S z-T z\|)+a\left\|S z-S x_{n}\right\|_{1}\right]}{1+M\|S z-T z\|}\right. \\
\leq & \left(1-\beta_{n}+a \beta_{n}\right)\left\|S x_{n}-p\right\|
\end{aligned}
$$

By using (4.2) in (4.1) becomes

$$
\begin{aligned}
\| S x_{n+1}- & p\left\|\leq\left(1-\alpha_{n}\right) a\right\| S x_{n}-p \| \\
& +\alpha_{n} a\left[\left\|\left(1-\beta_{n}\right)\left(p-S x_{n}\right)+\beta_{n}\left(T z-T x_{n}\right)\right\|\right] \\
\leq & \left(1-\alpha_{n}\right) a\left\|S x_{n}-p\right\| \\
& +\alpha_{n} a\left[1-\beta_{n}+a \beta_{n}\right]\left\|S x_{n}-p\right\| \\
= & a\left[1-(1-a) \alpha_{n} \beta_{n}\right]\left\|S x_{n}-p\right\| \\
\leq & {\left[1-(1-a) \alpha_{n}\right]\left\|S x_{n}-p\right\| } \\
\leq & \prod_{k=0}^{n}\left[1-(1-a) \alpha_{k}\right]\left\|S x_{0}-p\right\| \\
\leq & \prod_{k=0}^{n} e^{-(1-a) \alpha_{k}}\left\|S x_{0}-p\right\| \\
\leq & e^{-\left[(1-a) \sum_{k=0}^{n} \alpha_{k}\right]}\left\|S x_{0}-p\right\| \rightarrow 0 \text { as } n \rightarrow \infty
\end{aligned}
$$

Since $\sum_{k=0}^{\infty} \alpha_{k}=\infty$ and $\mathrm{a} \in[0,1)$. Hence, we obtain from (4.3) that $\left\|S x_{n}-p\right\| \rightarrow 0$ as $n \rightarrow \infty$, that is, $\left\{S x_{n}\right\}_{n=0}^{\infty}$ converges strongly to $\mathrm{p}$.

\section{Experiment}

To highlight the practical implication, we apply the iteration to solve the following equation

$$
x^{3}+4 x^{2}-5 x-10=0
$$

and prove that Jungck-Agarwal et al. iteration converges faster than Jungck-Noor, Jungck-Mann and Jungck-Ishikawa used by Bhagwati and Ritu [19] . We solve the equation by rewriting as by Bhagwati and Ritu [19]

$$
S x=x \text { and } T x=x^{3}+4 x^{2}-10
$$

Then the coincidence point of $\mathrm{S}$ and $\mathrm{T}$ in (5.2) leads to the solution of cubic equation (5.1). We compile and execute separate program in $\mathrm{C}++$ and show our output in table 1 by taking initial approximation

$$
x_{0}=1 \text { and } \alpha_{n}=\beta_{n}=\gamma_{n}=0.9
$$


Table-1

\begin{tabular}{|c|c|c|c|c|c|c|c|c|c|c|c|c|}
\hline \multicolumn{4}{|c|}{$\begin{array}{l}\text { Jungck-Agarwal et al. } \\
\text { Iteration Scheme }\end{array}$} & \multicolumn{3}{|c|}{$\begin{array}{l}\text { Jungck-Noor Iteration } \\
\text { Scheme }\end{array}$} & \multicolumn{3}{|c|}{ Jungck-Mann Iteration Scheme } & \multicolumn{3}{|c|}{$\begin{array}{l}\text { Jungck-Ishikawa Iteration } \\
\text { Scheme }\end{array}$} \\
\hline 0 & \begin{tabular}{|l|}
-7.6568 \\
\end{tabular} & \begin{tabular}{|l|}
-5 \\
\end{tabular} & -1.53136 & -4.24281 & \begin{tabular}{l|}
-5 \\
\end{tabular} & -0.848561 & -4 & -5 & -0.8 & -6.6568 & -5 & -1.33136 \\
\hline 1 & -7.11342 & -4.21088 & -1.42268 & \begin{tabular}{|l|}
-7.18707 \\
\end{tabular} & $\begin{array}{l}-7.73079 \\
\end{array}$ & -1.43741 & -7.5568 & -7.952 & -1.51136 & -6.59253 & -5.26978 & -1.31851 \\
\hline 2 & -6.76354 & -4.78344 & -1.35271 & -5.0744 & -4.70529 & -1.01488 & -4.63956 & -4.31543 & -0.927913 & -6.53485 & -5.33834 & -1.30697 \\
\hline 3 & -6.52719 & -5.15593 & -1.30544 & -6.65334 & -6.92539 & -1.33067 & -7.08334 & -7.35487 & -1.41667 & -6.48296 & -5.39985 & -1.29659 \\
\hline 4 & -6.36414 & -5.40802 & -1.27283 & \begin{tabular}{|l|}
-5.47281 \\
\end{tabular} & -5.27347 & -1.09456 & -5.04219 & -4.81539 & -1.00844 & -6.43619 & -5.45517 & -1.28724 \\
\hline 5 & -6.25059 & -5.58173 & --1.25012 & -6.36812 & -6.51909 & -1.27362 & -6.76619 & -6.95774 & -1.35324 & -6.39397 & -5.505 & -1.27879 \\
\hline 41 & \begin{tabular}{|l|}
-584443 \\
\end{tabular} & -5.98443 & -1.19689 & -5.98442 & -5.98441 & -1.19688 & -5.98665 & -5.98719 & -1.19733 & -5.99572 & -5.9713 & -1.19914 \\
\hline 42 & \begin{tabular}{|l|}
-5.98443 \\
\end{tabular} & -5.98443 & -1.19689 & -5.98445 & -5.98445 & -1.19689 & -5.98255 & -5.98209 & -1.19651 & -5.99468 & -5.97252 & -1.19894 \\
\hline 43 & -5.98443 & -5.98443 & -1.19689 & -5.98442 & -5.98442 & -1.19688 & -5.98604 & -5.98642 & -1.19721 & -5.99373 & -5.97362 & -1.19875 \\
\hline 51 & & & & \begin{tabular}{|c|}
-588443 \\
\end{tabular} & \begin{tabular}{|l|}
-5.98443 \\
\end{tabular} & $\begin{array}{l}-1.19689 \\
\end{array}$ & -5.98487 & -5.98498 & -1.19697 & -5.98871 & -5.97946 & -1.19774 \\
\hline 52 & & & & -5.98443 & \begin{tabular}{|l|}
-5.98443 \\
\end{tabular} & $\begin{array}{l}-1.19689 \\
\end{array}$ & -5.98406 & -5.98397 & -1.19681 & -5.98831 & $\begin{array}{l}-5.97992 \\
\end{array}$ & -1.19766 \\
\hline 53 & & & & -5.98443 & -5.98443 & -1.19689 & -5.98475 & -5.98483 & -1.19695 & -5.98795 & -5.98034 & -1.19759 \\
\hline 88 & & & & & & & -5.98443 & -5.98443 & -1.19689 & -5.98455 & -5.9843 & -1.19691 \\
\hline 89 & & & & & & & -5.98443 & -5.98443 & -1.19689 & -5.98454 & -5.98431 & -1.19691 \\
\hline 90 & & & & & & & -5.98443 & -5.98443 & -1.19689 & -5.98453 & -5.98432 & -1.19691 \\
\hline 138 & & & & & & & & & & $\begin{array}{l}-5.98443 \\
\end{array}$ & $\begin{array}{l}-5.98443 \\
\end{array}$ & $\begin{array}{l}-1.19689 \\
\end{array}$ \\
\hline 13 & & & & & & & & & & -5.98443 & -5.98443 & -1.19689 \\
\hline 140 & & & & & & & & & & -5.98443 & -5.98443 & $\begin{array}{l}-1.19689 \\
\end{array}$ \\
\hline
\end{tabular}

\section{Observation}

We observe that Jungck-Agarwal et al. iteration scheme converges faster than other Jungck Iteration schemes and decreasing order of convergence of Jungck iteration schemes is: Jungck-Agarwal et al., Jungck-Noor, Jungck-Mann, Jungck-Ishikawa.

\section{References}

[1.] Agarwal, R.P., O'Regan, D. and Sahu, D.R.: Iterative construction of fixed points of nearly asymptotically nonexpansive mappings, Journal of Nonlinear and Convex Analysis 8(1) (2007), 61-79.

[2.] Banach, S., Sur les Operations dans les Ensembles Abstraits et leur Applications aux Equations Integrals, Fund. Math. 3 (1922), 133-181

[3.] Berinde, V. : On the convergence of the Ishikawa iteration in the class of quasi-contractive operators, Acta Mathematica Universitatis Comenianae, vol. 73, no. 1, pp. 119-126(2004).

[4.] Berinde, V.: Iterative approximation of fixed points. Editura Efemeride, Baia Mare, (2002).

[5.] Berinde, V.: On the stability of some fixed point procedures. Bul. S, tiint. Univ. Baia Mare Ser. B Fasc.

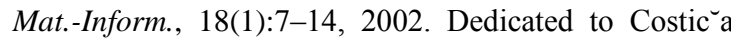
Must at, a on his 60th anniversary.

[6.] Harder, A. M. and Hicks, T. L.: Stability Results for Fixed Point Iteration Procedures, Math. Japonica 33 (5) (1988), 693-706.

[7.] Ishikawa, S., Fixed Point by a New Iteration Method, Proc. Amer. Math. Soc. 44 (1) (1974), 147-150

[8.] Jachymski, J. R.: An extension of A. Ostrowski's theorem on the round-off stability of iterations. Aequationes Math., 53(3):242-253, 1997.

[9.] Jungck, G.: Commuting mappings and fixed points, The American Mathematical Monthly, vol. 83, no.4, pp. 261263(1976).

[10.]Mann, W.R., Mean Value Methods in Iteration, Proc Amer. Math. Soc. 44 (1953), 506-510

[11.]Noor, M. A.: New approximation schemes for general variational inequalities, Journal of Mathematical Analysis and Applications, vol. 251, no. 1, pp. 217 229(2000).

[12.]Olatinwo , M. O. and Imoru, C. O.: Some convergence results for the Jungck-Mann and the Jungck-Ishikawa iteration processes in the class of generalized 
Zamfirescu operators, Acta Mathematica Universitatis Comenianae, vol. 77, no. 2, pp. 299-304( 2008).

[13.]Olatinwo, M. O.: Some stability and strong convergence results for the Jungck-Ishikawa iteration process, Creative Mathematics and Informatics, vol. 17, pp. 3342(2008).

[14.] Olatinwo, M. O.: A generalization of some convergence results using the Jungck-Noor three-step iteration process in an arbitrary Banach space, Fasciculi Mathematici, no. 40, pp. 37-43(2008).

[15.]Osilike, M. O. and Udomene, A.: Short proofs of stability results for fixed point iteration procedures for a class of contractive-type mappings. Indian J. Pure Appl. Math., 30(12):1229-1234, (1999).

[16.]Osilike, M. O.: Stability results for fixed point iteration procedures. J. Nigerian Math.Soc., 14/15:17-29, $(1995 / 96)$.

[17.]Osilike, M. O.: Stability results for the Ishikawa fixed point iteration procedure, Indian Journal of Pure and Applied Mathematics, vol. 26, no. 10, pp. 937-945 (1995)

[18.] Ostrowski, M. The round-off stability of iterations. $Z$. Angew. Math. Mech., 47:77-81, (1967).
[19.]P., Bhagwati and S., Ritu: Weak stability results for Jungck-Ishikawa iteration, International Journal of Computer Applications, Volume16, No. 4, February (2011).

[20.]Rhoades, B.E.: Fixed point theorems and stability results for fixed point iteration procedures. Indian J. Pure Appl. Math., 21(1):1-9, (1990).

[21.]Rhoades, B.E.: Fixed point theorems and stability results for fixed point iteration procedures. II. Indian J. Pure Appl. Math., 24(11):691-703, (1993).

[22.]Rhoades, B.E., Fixed Point Iteration using Infinite Matrices, Trans. Amer. Math. Soc. 196 (1974), 161176

[23.]Rhoades, B. E.: Comments on two fixed point iteration methods," Journal of Mathematical Analysis and Applications, vol. 56, no. 3, pp. 741-750(1976).

[24.]Singh, S. L. , Bhatnagar, Charu and Mishra, S. N.: Stability of Jungck-type iterative procedures International Journal of Mathematics and Mathematical Sciences, no. 19, pp. 3035-3043 (2005).

[25.]Zamfirescu, T., Fixed Point Theorems in Metric Spaces, Arch.Math.23(1972), 292-29. 